\title{
Editorial
}

\section{Michael Patriksson}

Received: 2 June 2010 / Accepted: 8 August 2010 / Published online: 24 August 2010

(C) Springer Science+Business Media, LLC 2010

The present special issue is the result of the workshop "Robust Multiobjective Design Optimization With Simulation", which was held at the Fraunhofer-Chalmers Research Centre for Industrial Mathematics (FCC), Gothenburg, Sweden, during December 3-4, 2007.

The subject of this workshop was robust optimal design where simulation is an important part of the design evaluation, and where there may be more than one objective function. The workshop gathered people from different fields, primarily from the Nordic countries, within academia and industry to discuss the state-of-the-art and future challenges in solving these problems. The scientific program consisted of plenary speeches by John Bandler (McMaster University, Toronto), Bo Bergman (Chalmers University of Technology, Gothenburg), Olof Dahlberg (CD-adapco, Seattle, WA), Anders Klarbring (Linköping University, Linköping), Karl-Heinz Küfer (ITWM, Kaiserslautern), and Per Weinerfelt (SAAB, Linköping), nine regular talks, and a final panel discussion containing stimulating discussions across subject boundaries on the future of the subject.

The first paper, by Jakobsson et al., develops a new, general approach to the global optimization of expensive and noisy black box functions, using a surrogate model based on radial basis functions (RBFs). A method for RBF-based approximation is introduced in order to handle noise, and new points are selected to minimize the total model uncertainty weighted against the surrogate function value. The algorithm is extended to multiple objective functions by weighting against the distance to the surrogate Pareto front, and therefore constitutes the first algorithm for expensive, noisy and multiobjective problems in the literature. Numerical results on analytical test functions, as well as from a simulation based optimization problem, show promise. 
The second paper, by Jakobsson et al., utilizes the algorithm from the first paper in the design of a diesel combustion engine. It is shown that good designs can be found that compromise between a low fuel consumption with low emissions of soot and nitrogen oxides.

The third paper, by Amoignon, describes the numerical platform AESOP for large scale aerodynamic shape optimization. It is built upon the adjoint (primal-dual) method in computational fluid dynamics, as applied to the complete system including parameterization, mesh deformation, the primal-to-dual mesh transformation and the flow equations, solved by the unstructured flow solver Edge distributed by FOI. The numerical platform integrates this solver with algorithms of shape parameterization and gradient-based descent methods. Application examples are given, and a summary is also provided of how RBFs can be profitably used in the shape parameterization.

The fourth paper, by Colson et al., is founded on the recognition that advanced nonlinear analyses developed for estimating structural responses for recent applications for the aerospace industry lead to expensive computations. The authors compare several numerical optimization approaches, including sequential convex programming, derivative free optimization, surrogate based optimization, and genetic algorithms, for the optimal design of stiffened aircraft panels with respect to local and global instabilities (buckling and collapse). The computations are carried out with software developed for the European aeronautical industry.

The fifth and last paper, by Wadbro, considers the imaging technique known as microwave tomography, especially for medical applications. The paper provides a reconstruction procedure using a sequence of problems solved by topology optimization techniques, where each problem is constructed based on the current solution and a priori information on the (unknown) permittivities. Numerical examples illustrate the reconstruction procedure for the case when the sizes of the objects are of the same order as the wavelength. A good estimate of the permittivities is obtained using a priori information that the unknown permittivities vary within a given box and a bound on how lossy the unknown objects are.

The workshop was organized and partly sponsored by GMMC (Gothenburg Mathematical Modelling Centre) which is a research centre placed at the Department of Mathematical Sciences (Chalmers and University of Gothenburg) and FCC (see http://www.chalmers.se/math/EN/research/gmmc); it is a strategic research centre funded by the Swedish Foundation for Strategic Research (SSF). Their sponsorship of the workshop is greatly appreciated. For assistance with the workshop's scientific program, Dr. Ann-Brith Strömberg, FCC, and Dr. Fredrik Edelvik, FCC, deserve special mention; for assistance during the workshop a special thanks goes to Lina Säfström, FCC. The diligent work of the referees are also greatfully acknowledged.

Göteborg, September 2009 


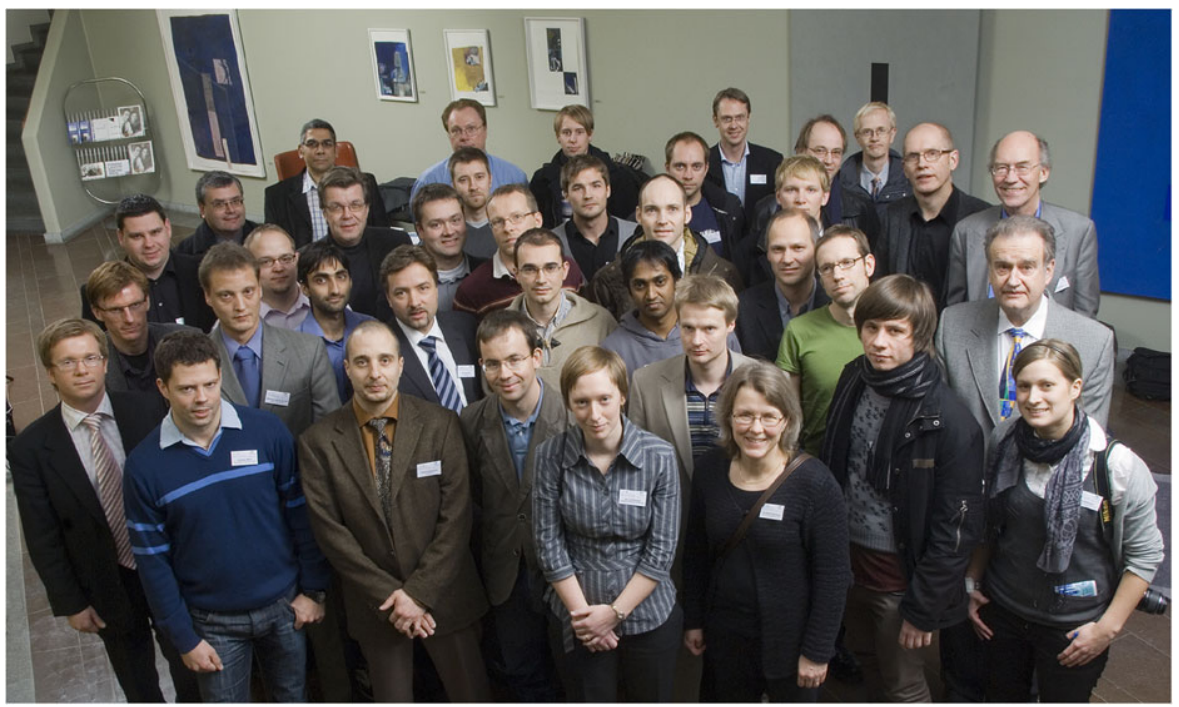

Photograph taken by Jan-Olof Yxell. 